\title{
ESEM Study of Magnetic Bubbles - Surface Morphological and Fluidic Aspects
}

\author{
Zahava Barkay ${ }^{1}, \mathrm{Yu} \mathrm{Gao}{ }^{2}$, Chenjie $\mathrm{Xu}^{2}$ and Claus-Dieter Ohl ${ }^{3}$ \\ 1. Wolfson Applied Materials Research Center, Tel-Aviv University, Tel-Aviv 6997801, Israel \\ 2. Division of Bioengineering, School of Chemical and Biomedical Engineering, Nanyang Technological \\ University, Singapore 637457, Singapore \\ 3. Division of Physics and Applied Physics, School of Physical and Mathematical Sciences, Nanyang \\ Technological University, Singapore 637371, Singapore
}

Liquid droplets encapsulated by hydrophobic powder, i.e. liquid marbles, were intensively studied over the last few decades due to their unique physical properties and applications [1]. Alternative structures of gas bubbles with nanoparticle shell have recently gained much attention for their compression properties and potential usage in a large variety of biological applications. The current work has focused on magnetic microbubbles with self-assembled nanoparticle shell for drug delivery applications. The preparation of similar magnetic bubbles was previously reported and their dynamics and manipulation in acoustic and magnetic fields was explored using optical microscopy [2].

The current study uses multiple modes of high resolution electron microscopy at wet and dry environmental conditions for characterization of innovative magnetic microbubbles with iron and silica self-assembled nanoparticle shell [2-3]. The results include: surface morphology and composition of bubble shell, characterization of bubble self-assembly, bubble stability under various environmental conditions, and in-situ dynamic imaging of bubble flow including triple line characterization. The imaging was carried out in a controlled temperature-pressure environment using Quanta 200FEG environmental scanning electron microscope (ESEM) as shown elsewhere [4].

Bubble saturated liquid droplets were examined in ESEM wet mode following a dehydration process for evaluating the bubble stability during liquid evaporation. The analysis of bubbles after dehydration was based on comparing the secondary electron (SE) mode, which provided surface morphology at various sample tilt angles, with the scanning transmission electron microscopy (STEM) mode [5]. The agglomeration of microbubbles (each of $4.2 \pm 0.9 \mu \mathrm{m}$ size) into chain-like shapes of tens micron length, provided indication for self-assembled bubble process in addition to the known self-assembly of shell particles (Figs. 1a-c). The existence of both silica and iron particles on the bubble surface was verified by EDS (energy dispersive spectroscopy) analysis. The size was $39 \pm 9 \mathrm{~nm}$ and $340 \pm 80 \mathrm{~nm}$ respectively for iron and silica shell particles and their relative surface arrangement was thus revealed (Figs. 1d-f).

In-situ dynamic wet-mode ESEM imaging of bubble flow within the droplet showed that bubble selfassembly occurred already at the liquid state before dehydration. The bubbles were transported outward the droplet, which facilitated their self-assembly at the droplet edge as could be expected by the coffee ring effect [6]. Some bubbles were popping out of the droplet surface as shown at Fig. 2a, while most bubbles remained at the liquid volume and thus were revealed after liquid evaporation. The droplet triple line was examined showing bubble ejection out and air-pocket formation (Figs. 2b-c). The distortion of triple line shape and the inhomogeneous triple line pinning by bubbles were particularly significant at the droplet evaporation process. Previous ESEM research on liquid droplets on textured substrates showed similar air-pockets at the triple line [4]. However, the current study referred to droplets on smooth silicon wafer substrates and thus the distortion of triple line structure was attributed to the 
intrinsic bubble-liquid properties. The described ESEM study of the magnetic microbubbles referred to two related aspects (morphological and fluidic), which should be further quantitatively studied and considered for high efficiency targeted drug delivery and for lab-on-chip device applications [7].

\section{References:}

[1] P. G. de Gennes et al, "Capillarity and wetting phenomena", (Springer, NY) p.226.

[2] X. Zhao et al, Phys. Rev. Lett. 102 (2009), p. 024501.

[3] Y. Gao et al, J. Mater. Chem. B. in press (2015).

[4] E. Bormashenko et al, Langmuir 29 (2013), p. 14163.

[5] Z. Barkay et al, Micron 40 (2009), p. 480.

[6] R. D. Deegan et al, Nature 389 (1977), p. 827.

[7] The authors acknowledge the Materials and Nano centers at Tel-Aviv University for feasibility study at the ESEM laboratory.
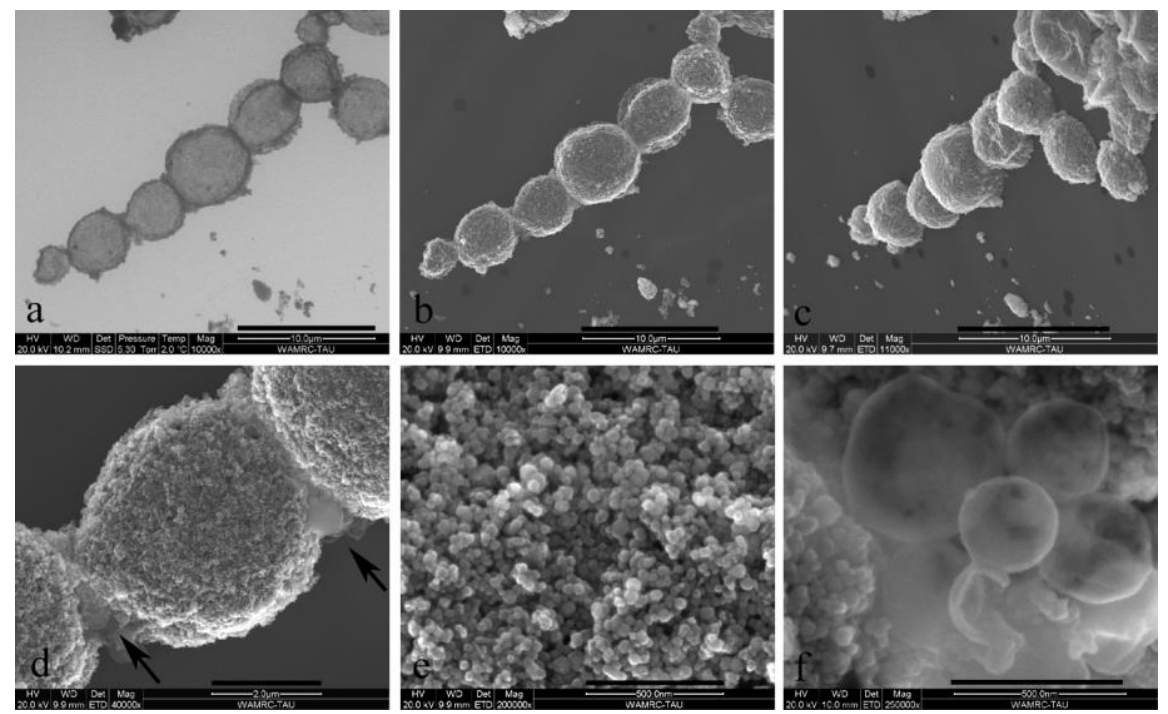

Figure 1. ESEM of self-assembled microbubbles: (a) bright-field STEM mode, (b) SE mode at zero tilt, (c) SE mode at $60^{\circ}$ tilt ((a)-(c) scale bar $\left.10 \mu \mathrm{m}\right)$, (d) a single bubble with silica particles indicated by an arrow (scale bar $2 \mu \mathrm{m}$ ), (e) iron particles, (f) silica particles ((e)-(f) scale bar 500nm).
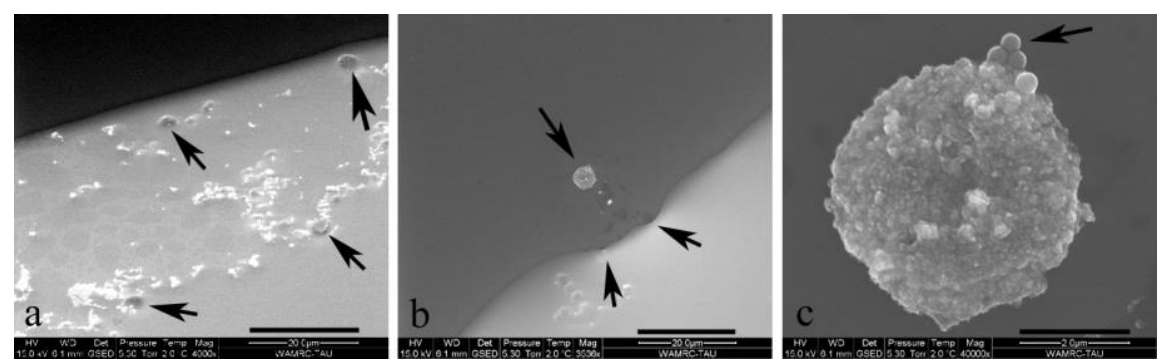

Figure 2. ESEM "wet-mode" in-situ dynamic study: (a) microbubbles popping out of the liquid drop (indicated by arrows, scale bar $20 \mu \mathrm{m}$ ), (b) a microbubble ejected (top arrow) and air-pockets at the triple line (two bottom arrows, scale bar $20 \mu \mathrm{m}$ ), (c) the microbubble after ejection with mainly iron particles and some silica particles on top (indicated by an arrow, scale bar $2 \mu \mathrm{m}$ ). 